\title{
Synthesis of Some C-5 Branched Hexopyranoses
}

\author{
Lars Kilaas $^{\mathrm{a}}$ and Thorleif Anthonsen ${ }^{\mathrm{b}}$ \\ a SINTEF, Section for Applied Chemistry, N-7034 Trondheim-NTH and ${ }^{\mathrm{b}}$ Department of Chemistry, The University of Trondheim, \\ $\mathrm{N}-7055$ Trondheim, Norway
}

\begin{abstract}
Kilaas, L. and Anthonsen, T., 1992. Synthesis of Some C-5 Branched Hexopyranoses. - Acta Chem. Scand. 46: 994-999.

The stereoselectivity of diazomethane and dimethylsulfonium methylide additions to the $\mathrm{C}-5$ aldehyde group in 1,2-O-isopropylidene-3-O-methyl- $\alpha$-D-xylo-pentodialdo-1,4furanose or the C-5 ketone function of 6-deoxy-1,2-O-isopropylidene-3- $O$-methyl$\alpha$-D-xylo-hexofuranos-5-ulose and 1,2-O-isopropylidene-3- $O$-methyl-5- $C$-phenyl- $\alpha$ D-xylo-pentofuranos-5-ulose have been investigated. Reactions with the aldehyde and the methyl ketone gave predominantly the gluco-epoxide while the ido-epoxide was the predominant product from the phenyl ketone. During the reactions the following pyranoses were isolated and characterized: 1,6-anhydro-3- $O, 5$ - $C$-dimethyl$\beta$-L-idopyranose, 1,6-anhydro-3- $O$-methyl-5- $C$-phenyl- $\beta$-L-idopyranose, 3- $O, 5$ - $C$-dimethyl-D-glucopyranose and 1,6-anhydro-3- $O, 5$ - $C$-dimethyl- $\beta$-D-glucopyranose.
\end{abstract}

Monosaccharides with methyl, hydroxymethyl and formyl branching have been isolated from plant sources. For instance apiose (3-C-hydroxymethyl-D-glycero-tetrulose) has been isolated from parsley, ${ }^{1}$ hamamelose $(2-C$-hydroxymethyl-D-ribose) from witch hazel, ${ }^{1}$ the alditol 2- $C$-methylD-erythritol from Convolvulus glomeratus ${ }^{2}$ and 2-C-methylD-erythronic acid from cacti. ${ }^{3}$ The interest in branched carbohydrates was greatest when they were constituents of antibiotics such as olivomycin (2,6-dideoxy-3- $C$-methyl-Larabino-hexose), ${ }^{1}$ streptomycin (5-deoxy-3-C-formyl-L-lyxose) ${ }^{1}$ and lancamycin (2,6-dideoxy-3-C, 3-O-dimethyl-Lxylo-hexose). ${ }^{1}$ The synthesis of such branched carbohydrates, which includes formation of chiral centres, has, to a great extent, been based upon appropriate oxo derivatives. The stereoselectivities obtained from different reactions have varied. ${ }^{4}$ For 4-uloses stereoselectivity of Grignard addition depends on the orientation of the C-3 oxygen function. In pyranose rings equatorial oxygen functions at $\mathrm{C}-3$ result preferentially in equatorial attack at $\mathrm{C}-4$, while the presence of axial C-3 functions gives the opposite orientation of the attack. 4,6-O-Benzylidene derivatives of a number of 2- and 3-uloses have been examined with respect to the stereoselectivity of Grignard reactions. While 3uloses show a strong preference for equatorial attack by methyl nucleophiles, reactions with the 2-uloses are less selective. $^{4}$

During investigations on the stereoselectivity of additions of diazomethane to some exocyclic ketones with chiral $\alpha$-carbon atoms, we have synthesized a number of carbohydrates with $\mathrm{C}-5$ branching.

\section{Results and discussion}

The starting point for the reactions was $1,2-O$-isopropylidene-3-O-methyl- $\alpha$-D-glucofuranose $(1)^{5}$ which is readily obtained from D-glucose. Oxidation of 1 with sodium periodate gave 1,2-O-isopropylidene-3- $O$-methyl- $\alpha$-D-xylopentodialdo-1,4-furanose (2). ${ }^{6}$ We have previously investigated the stereoselectivity of Grignard additions to this compound and we have been able to show that it is possible to influence the ratio of the products due to chelation and non-chelation mechanisms by utilizing pentaoxapentadecane as a competing chelator. ${ }^{7} 1,2-O$-isopropylidene- $\alpha$-Dxylo-pentodialdo-1,4-furanose lacking the 3-O-methyl group has also been studied. This compound exists as a dimer whose structure has been proved to change on going from chloroform to methanol as the solvent. ${ }^{8}$

Although additions to the oxo group in aldehydes will not produce a branching point, it is of general interest to investigate the stereoselectivity of the reactions and to characterize new products. Addition of diazomethane in diethyl ether to 2 gave the two diastereomeric epoxides $3: 4$ in the ratio $75: 25$. This ratio changed slightly to $83: 17$ in a reaction medium containing $40 \%$ methanol. Similar solvent effects have been shown previously. ${ }^{9}$

It is well known that addition of diazomethane to ketones also leads to formation of ketones with an additional carbon atom in the chain instead of a branch. ${ }^{10}$ This was also observed in our reactions in varying amounts. The diastereomeric epoxides were separated by TLC. The NMR spectra of these products were very similar and direct identification from the spectra of the two products was not possible. Tosylation of 1 gave both the monotosylate 5 and the ditosylate 6 which were separated. The point of tosylation in 5 was inferred from the ${ }^{1} \mathrm{H}$ NMR shift of $\mathrm{H}_{5}$ which appeared at $4.25 \mathrm{ppm}$. This is close to the value of the corresponding proton in $1(4.02 \mathrm{ppm})$. The other monotosylate was also observed in minor amounts and the shift of $\mathrm{H}_{5}$ here was $5.11 \mathrm{ppm}$. Treatment of 5 with sodium methoxide in methanol gave 3 in high yield. This is a normal $S_{N} 2$ 


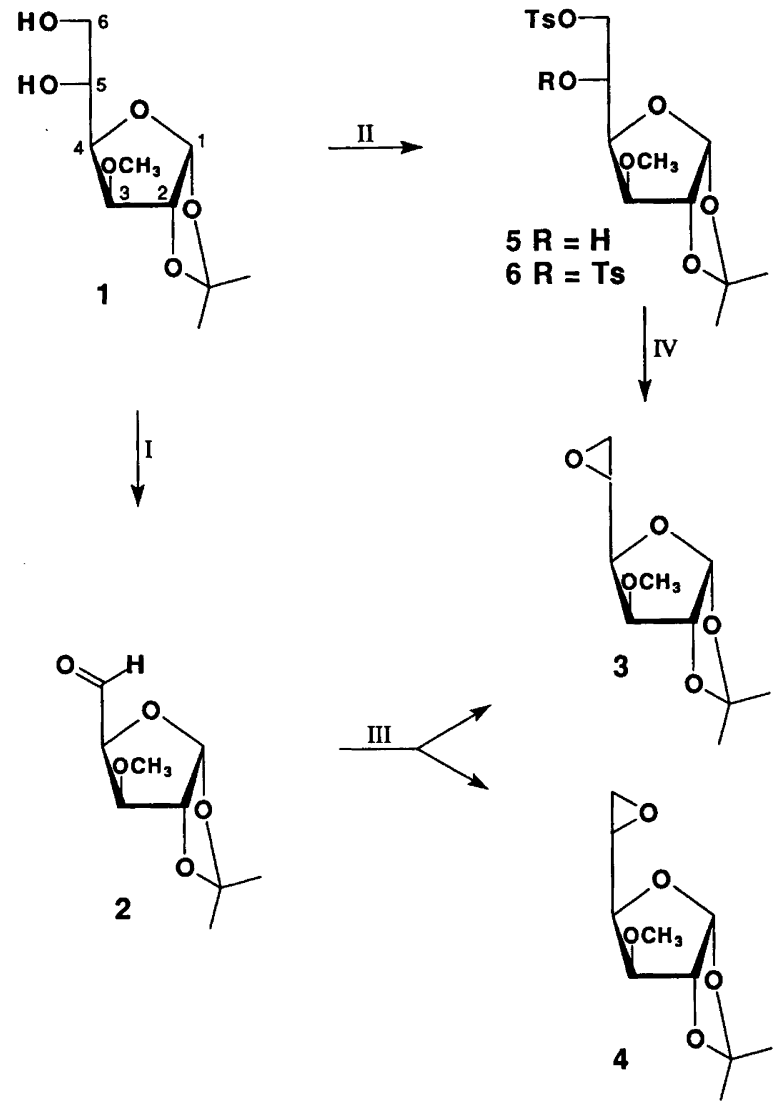

Scheme 1. Reaction conditions: I, $\mathrm{NaIO}_{4} / \mathrm{H}_{2} \mathrm{O}$; II, $p$-TosCl/ $\mathrm{CHCl}_{3}$; III, (a) $\mathrm{Me}_{2} \mathrm{SCH}_{2} / \mathrm{DMSO}$, (b) $\mathrm{CH}_{2} \mathrm{~N}_{2} / \mathrm{Et}_{2} \mathrm{O}$; IV, $\mathrm{Na} / \mathrm{MeOH}$.

reaction which does not influence the stereochemistry at $\mathrm{C}_{5}$. Consequently, this reaction proves that the predominant product of the diazomethane addition reaction to 1 was the D-gluco-isomer 3 and strongly implies that 4 was the L-idofuranose (Scheme 1).

It is a well established technique to use sulfur ylides to produce epoxides by addition reactions. ${ }^{11}$ Addition of dimethylsulfonium methylide to the aldehyde 2 gave an even higher excess of 3 , the ratio being $3: 4=86: 14$. The isolated yield of 3 in this reaction was $43 \%$. In contrast with aldehydes, addition of carbon nucleophiles to carbohydrate ketones will create branched carbohydrates. The methyl ketone 6-deoxy-1,2-O-isopropylidene-3- $O$-methyl- $\alpha$-D- $x y l o-$ hexofuranos-5-ulose (7) was produced by a Grignard reaction followed by ruthenium tetraoxide oxidation (Scheme 2). Addition of diazomethane in diethyl ether to 7 gave a mixture of epoxides 8 and 9 in a ratio of $40: 60$. Addition of diazomethane in a diethyl ether:methanol solution $(2: 1)$ reversed the ratio of $8: 9$ to $55: 45$. The two epoxides were separated on an $\mathrm{SiO}_{2}$ column, isolated in a total yield of $60 \%$, and their structures were determined after subsequent base-catalysed opening of the epoxide ring and acidcatalysed removal of the 1,2-O-isopropylidene group (Scheme 3). The minor product epoxide yielded 12 via 10 while the predominant product yielded a mixture of $\mathbf{1 3}$ and 14 via 11. Consequently, the ratio of the epoxides $8: 9$ was
40:60. The structures of the key compounds 12,13 and 14 were verified by ${ }^{1} \mathrm{H}$ NMR spectroscopy. Acid treatment of 5- $C$-methyl-1,2- $O$-isopropylidene-3- $O$-methyl- $\beta$-L-idofuranose (10) led spontaneously to the formation of 1,6-anhydro-3- $O, 5$ - $C$-dimethyl- $\beta$-L-idopyranose (12). In this structure both the C-5 methyl group and the three oxygen functions at $\mathrm{C}-2, \mathrm{C}-3$ and $\mathrm{C}-4$ are in equatorial positions and the conformation is ${ }^{4} \mathrm{C}_{1}(\mathrm{~L}) .{ }^{12}$ The vicinal coupling constants agree with the structure. An additional proof of the 1,6-anhydro structure is provided by the presence of a ${ }^{4} \mathrm{~J}$ coupling of $1.4 \mathrm{~Hz}$ between $\mathrm{H}-4$ and $\mathrm{H}-6($ exo $)$ indicating a rigid structure where the bonds joining the two mentioned protons form a planar $\mathrm{W}$-structure.

The hydrolysis of 1,2-O-isopropylidene-3-O,5-C-dimethyl- $\alpha$-D-glucofuranose (11) also gave a 1,6-anhydro compound 14, but in about equal amounts to the monocyclic sugar 13. The reason for this different behaviour is quite obvious. In the structure of 1,6-anhydro-3-O, $5-\mathrm{C}$ dimethyl- $\beta$-D-glucopyranose (14), although the C-5 methyl group is equatorially oriented, the three oxygen functions at $\mathrm{C}-2, \mathrm{C}-3$ and $\mathrm{C}-4$ are all in axial positions. In the ${ }^{1} \mathrm{H}$ NMR spectrum of 14 , all the vicinal couplings are very small and furthermore there is no long-distance coupling as in the case of 12. This is due to the absence of the $W$ structure. The conformation of 14 is ${ }^{1} C_{4}(D)$.

In contrast, the product not having anhydro structure, 3-O, 5-C-dimethyl- $\alpha$-D-glucopyranose (13) showed large vicinal couplings $\left(J_{2,3}\right.$ and $\left.J_{3,4}=9.3 \mathrm{~Hz}\right)$ indicating axial protons at C-2, C-3 and C-4. Thus, this compound is in the ${ }^{4} \mathrm{C}_{1}(\mathrm{D})$ conformation with the C-5 methyl group axially oriented. The anomeric ratio as determined by ${ }^{1} \mathrm{H}$ NMR spectroscopy in $\mathrm{D}_{2} \mathrm{O}$ was $\alpha: \beta=15: 85$. Owing to the presence of the axial C-5 methyl group, the $\beta$-anomer is even more predominant than for D-glucopyranose $(\alpha: \beta=$ $38: 62)$.

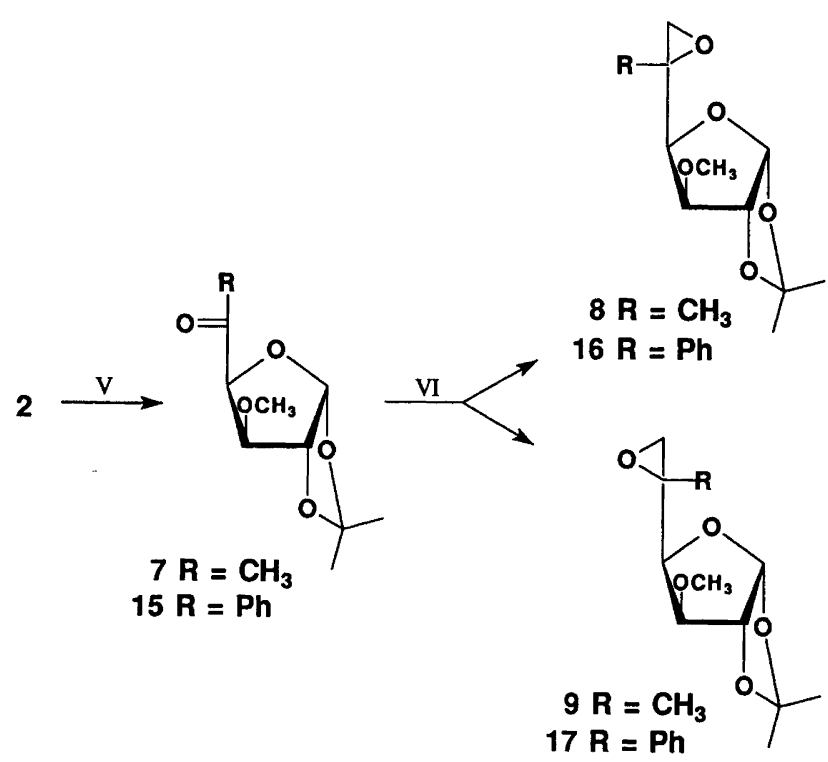

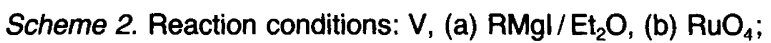
$\mathrm{VI}, \mathrm{CH}_{2} \mathrm{~N}_{2} / \mathrm{Et}_{2} \mathrm{O}$. 

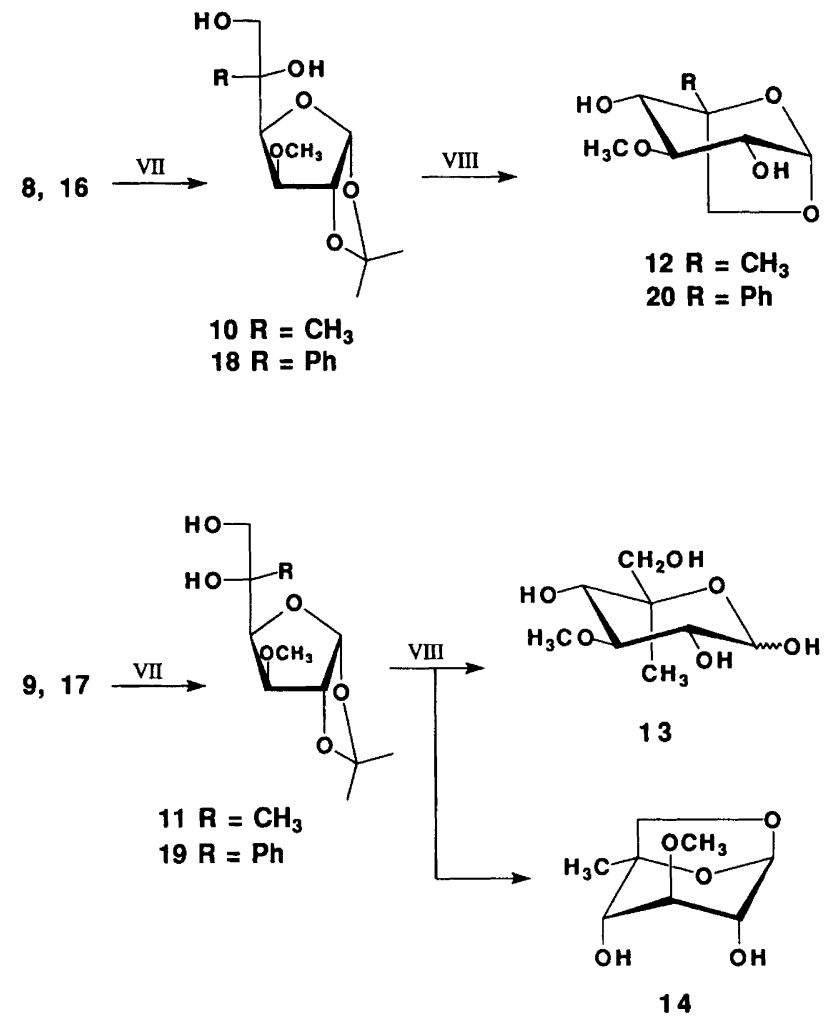

Scheme 3. Reaction conditions: VII, $0.5 \mathrm{M} \mathrm{NaOH}$; VIII, 0.8-2\% $\mathrm{H}_{2} \mathrm{SO}_{4}$.

Starting with 2 the phenyl ketone 15 was made in the same manner as 7 . When 15 was reacted with diazomethane in diethyl ether the two epoxides 16 and 17 were formed in the ratio $16: 17=89: 11$. The structure of 16 was proved by the same sequence of base- and acid-hydrolysis as previously described for $8 .{ }^{1} \mathrm{H}$ and ${ }^{13} \mathrm{C}$ NMR data for 18 were clearly seen from the spectra of the mixture of 18 and 19. Purification was performed in the last step and this yielded $\quad 1,6$-anhydro-3- $O$-methyl-5- $C$-phenyl- $\beta$-L-idopyranose (20) derived from 18 . The characteristic long-distance coupling between $\mathrm{H}-4$ and $\mathrm{H}-6($ exo $)$ for 20 was observed $\left({ }^{4} \mathrm{~J}\right.$ $=0.9 \mathrm{~Hz}$ ). The reactions with 15 gave an excess of the ido-epimer which is the opposite of the reactions with $\mathbf{2}$ and 7. The diazomethane additions were performed in diethyl ether with varying amounts of methanol. Homologation, which is a competing reaction to epoxide formation, was favoured with increasing methanol concentration. As has been suggested previously ${ }^{9}$ homologation and formation of the gluco-epoxide may share a common intermediate.

\section{Experimental}

All chemicals were of pro analysi grade and the solvents were dried. Diazomethane was prepared from $N$-methyl$N$-nitrosotoluene- $p$-sulphonamide. ${ }^{13}$ Dimethylsulfonium methylide was made according to Corey and Chaykovsky. ${ }^{11}$ ${ }^{1} \mathrm{H}$ NMR spectra were recorded at $400 \mathrm{MHz}$ on a Bruker WM-400 instrument and ${ }^{13} \mathrm{C}$ spectra at $25.1 \mathrm{MHz}$ on a Jeol
FX-100 instrument in $\mathrm{CDCl}_{3}$ solutions unless otherwise stated. Optical rotations were measured with a Perkin Elmer 241 polarimeter. Mass spectroscopy was performed on an AEI MS902 double focussing spectrometer using the direct inlet probe and electron impact at $70 \mathrm{eV}$. Both thin layer chromatography (TLC) and preparative layer chromatography (PLC) were performed on Merck fertigplatten (Silica gel $\mathrm{G} 60 \mathrm{~F}_{254}, 0.25 \mathrm{~mm}$ ). Medium-pressure chromatography was run on $30 \mathrm{~cm}$ columns using Merck Silica gel 60,400 mesh and $4 \mathrm{~atm}$ pressure provided by a Duramat pump. For gas chromatography a Varian 3400 instrument with split-splitless injector, FID and equipped with a Vista 402 data system were used. Hydrogen was used as the carrier gas and the columns were BP-1 (cross-linked methyl silicon) and SP-2100 (methyl silicon oil) both $25 \mathrm{~m} \times 0.33$ $\mathrm{mm}, 0.25 \mu \mathrm{m}$. 1,2-O-Isopropylidene-3- $O$-methyl- $\alpha$-D-xylopentodialdo-1,4-furanose (2) was made from 1,2-O-isopropylidene-3- $O$-methyl- $\alpha$-D-glucofuranose $(1)^{5}$ [CAS reg. No. 43138-65-4] according to the general procedure by Guthrie. $^{6}$

Reaction of $\mathbf{2}$ with dimethylsulfonium methylide in DMSO/THF. A solution of $2(0.2 \mathrm{~g}, 0.99 \mathrm{mmol})$ in THF $(10 \mathrm{ml})$ under $\mathrm{N}_{2}$ was added dropwise to a suspension of dimethylsulfonium methylide $(1.5 \mathrm{ml}, 1.2 \mathrm{mmol})$. After the reaction mixture had been stirred at $0^{\circ} \mathrm{C}$ for $20 \mathrm{~min}$ and subsequently at room temperature for $1.75 \mathrm{~h}, \mathrm{H}_{2} \mathrm{O}(50 \mathrm{ml})$ was added and the reaction mixture extracted with $\mathrm{Et}_{2} \mathrm{O}$ $(3 \times 100 \mathrm{ml})$. Work-up yielded a light syrup $(0.2 \mathrm{~g}, 0.93$ mmol, $93 \%$ ) which contained 3 and 4 in the ratio $86: 14$. PLC of a part of the mixture $(80 \mathrm{mg})$ using $\mathrm{CHCl}_{3}$ as the eluent gave pure $\left(R_{\mathrm{f}}=0.64\right)$ 5,6-anhydro-1,2-O-isopropylidene-3- $O$-methyl- $\alpha$-D-glucofuranose (3) $(40 \mathrm{mg}, 43 \%)$ as an oil. ${ }^{1} \mathrm{H}$ NMR: $\delta 5.92\left(\mathrm{H}-1, \mathrm{~d}, J_{1,2}=3.7 \mathrm{~Hz}\right), 4.62(\mathrm{H}-2$, $\left.\mathrm{d}, J_{2,3}=0 \mathrm{~Hz}\right), 3.85\left(\mathrm{H}-3, \mathrm{~d}, J_{3,4}=3.0 \mathrm{~Hz}\right), 3.73(\mathrm{H}-4$, dd, $\left.J_{4,5}=7.1 \mathrm{~Hz}\right), 3.24\left(\mathrm{H}-5, \mathrm{ddd}, J_{5,6 \mathrm{a}(c i s)}=2.5 \mathrm{~Hz}, J_{5,6 \mathrm{~b}(\text { trans })}=\right.$ $4.0 \mathrm{~Hz}), 2.92\left(\mathrm{H}-6 \mathrm{~b}, \mathrm{dd}, J_{\text {gem }}=5.0 \mathrm{~Hz}\right), 2.78(\mathrm{H}-6 \mathrm{a}, \mathrm{dd})$, $3.50\left(\mathrm{OCH}_{3}, \mathrm{~s}\right), 1.47\left[\mathrm{CH}_{3}\right.$, isopropyl (endo), s], $1.32\left(\mathrm{CH}_{3}\right.$, isopropyl (exo), s]. ${ }^{13} \mathrm{C}$ NMR: $\delta 105.2(\mathrm{C}-1, \mathrm{~d}), 82.0(\mathrm{C}-2$, d), $84.3(\mathrm{C}-3, \mathrm{~d}), 81.5\left(\mathrm{C}_{4}, \mathrm{~d}\right), 47.9(\mathrm{C}-5, \mathrm{~d}), 46.6(\mathrm{C}-6, \mathrm{t})$, $111.8\left(\right.$ ketal C, s), 58.2 $\left(\mathrm{OCH}_{3}, \mathrm{q}\right) 26.1$ and $16.6\left(2 \times \mathrm{CH}_{3}\right.$, isopropyl, q). Fractions with $R_{\mathrm{f}}=0.5$ contained 5,6-anhydro-1,2- $O$-isopropylidene-3- $O$-methyl- $\beta$-L-idofuranose (4) $(8.5 \mathrm{mg}, 9 \%)$. ${ }^{1} \mathrm{H}$ NMR: $5.97\left(\mathrm{H}-1, \mathrm{~d}, J_{1,2}=3.7 \mathrm{~Hz}\right)$, $4.62\left(\mathrm{H}-2, \mathrm{~d}, J_{2,3}=0 \mathrm{~Hz}\right), 3.79\left(\mathrm{H}-3, \mathrm{~d}, J_{3,4}=3.5 \mathrm{~Hz}\right), 3.85$ $\left(\mathrm{H}-4, \mathrm{dd}, J_{4,5}=5.7 \mathrm{~Hz}\right), 3.23\left(\mathrm{H}-5, \mathrm{ddd}, J_{5,6 a(c i s)}=2.7 \mathrm{~Hz}\right.$, $\left.J_{5,6 \mathrm{~b} \text { (rans) }}=4.4 \mathrm{~Hz}\right), 2.69\left(\mathrm{H}-6 \mathrm{a}, \mathrm{dd}, J_{\text {gem }}=4.8 \mathrm{~Hz}\right), 2.85$ (H-6b, dd), $3.45\left(\mathrm{OCH}_{3}, \mathrm{~s}\right), 1.47\left[\mathrm{CH}_{3}\right.$, isopropyl (endo), s], $1.33\left[\mathrm{CH}_{3}\right.$, isopropyl $($ exo $\left.), \mathrm{s}\right] \cdot{ }^{13} \mathrm{C}$ NMR: $105.4(\mathrm{C}-1, \mathrm{~d})$, 81.9 (C-2, d), 85.7 (C-3, d), 81.9 (C-4, d), 49.0 (C-5, d), $43.3(\mathrm{C}-6, \mathrm{t}), 111.8(\mathrm{ketal} \mathrm{C}, \mathrm{s}), 58.2\left(\mathrm{OCH}_{3}, \mathrm{q}\right), 26.4$ and $27.0\left(2 \times \mathrm{CH}_{3}\right.$, isopropyl, q).

Reaction of 2 with diazomethane. A 0.5 molar solution of $\mathrm{CH}_{2} \mathrm{~N}_{2}$ in $\mathrm{Et}_{2} \mathrm{O}(9 \mathrm{ml})$ was added dropwise to a solution of 2 $(250 \mathrm{mg}, 1.2 \mathrm{mmol})$ in dry $\mathrm{Et}_{2} \mathrm{O}(5 \mathrm{ml})$. When the reaction was complete (no more evolution of $\mathrm{N}_{2}$ ), the solvent was 
evaporated off under reduced pressure to yield a pale yellow syrup (259 mg, $1.2 \mathrm{mmol}$ ), 3:4 = 75:25 (GLC).

\section{1,2-O-Isopropylidene-3-O-methyl-6-O-tosyl- $\alpha$-D-glucofura-} nose (5). Under stirring at $0^{\circ} \mathrm{C}$ an ice-cold solution of $p$ - $\mathrm{TosCl}(5.5 \mathrm{~g}, 28.9 \mathrm{mmol})$ in $\mathrm{CHCl}_{3}(10 \mathrm{ml})$ was added to a suspension of $1(5.5 \mathrm{~g}, 23.5 \mathrm{mmol})$ in dry pyridine $(10 \mathrm{ml})$. After an additional $48 \mathrm{~h}$ of stirring, crushed ice was added and the mixture was extracted with $\mathrm{CHCl}_{3}(3 \times 50 \mathrm{ml})$. Work-up yielded a yellow syrup $(7.89 \mathrm{~g}) . \mathrm{CHCl}_{3}(15 \mathrm{ml})$ was added and the mixture was stored at $-20^{\circ} \mathrm{C}$ for $24 \mathrm{~h}$. Crystallized ditosylate 6, m.p. $118-118.5^{\circ} \mathrm{C}$, was removed by filtration. MS: Calcd. for $\mathrm{C}_{24} \mathrm{H}_{30} \mathrm{O}_{10} \mathrm{~S}_{2}=542.1280$. Obs. 542.1268. ${ }^{1} \mathrm{H}$ NMR: $\delta 5.76\left(\mathrm{H}-1, \mathrm{~d}, J_{1,2}=3.64 \mathrm{~Hz}\right), 4.53$ $\left(\mathrm{H}-2, \mathrm{~d}, J_{2,3}=0 \mathrm{~Hz}\right), 3.78\left(\mathrm{H}-3, \mathrm{~d}, J_{3,4}=3.12 \mathrm{~Hz}\right), 4.27$ $\left(\mathrm{H}-4, \mathrm{dd}, J_{4,5}=7.34 \mathrm{~Hz}\right), 5.07(\mathrm{H}-5, \mathrm{~m}), 4.04$ and 4.33 (H-6a and H-6b, resp., $2 \times \mathrm{dd}, J_{\text {gem }}=11.29 \mathrm{~Hz}, J_{5,6 \mathrm{a}}=6.24$ $\left.\mathrm{Hz}, J_{5,6 \mathrm{~b}}=2.07 \mathrm{~Hz}\right), 3.33\left(3 \mathrm{H}, \mathrm{OCH}_{3}, \mathrm{~s}\right), 1.30\left[3 \mathrm{H}, \mathrm{CH}_{3}\right.$, isopropyl (exo), s], $1.44\left[3 \mathrm{H}, \mathrm{CH}_{3}\right.$, isopropyl (endo), s], 7.79 and $7.65\left(2 \times 2 \mathrm{H}, \mathrm{H}-2^{\prime}, \mathrm{H}-6^{\prime}, \mathrm{d}, J=8.1 \mathrm{~Hz}\right), 7.34$ and $7.29\left(2 \times 2 \mathrm{H}, \mathrm{H}-3^{\prime}, \mathrm{H}-5^{\prime}, \mathrm{d}\right), 2.42\left(3 \mathrm{H}, \mathrm{CH}_{3}\right.$, tos $) .{ }^{13} \mathrm{C}$ NMR: $\delta 105.1$ (C-1, d), 80.6 (C-2, d), 82.5 (C-3, d), 78.0 (C-4, d), 68.5 (C-5, d), 74.7 (C-6, t), 112.2 (ketal C, s), 57.5 $\left(\mathrm{OCH}_{3}, \mathrm{q}\right), 26.2$ and $26.8\left(2 \times \mathrm{CH}_{3}\right.$, isopropyl, $\left.\mathrm{q}\right), 21.6$ $\left(2 \times \mathrm{CH}_{3}\right.$, tos, q), 144.9 and $144.7\left(2 \mathrm{C}-1^{\prime}, \mathrm{s}\right), 129.7,128.0$ (2 C-2', s and 2 C-3', s), 132.3 and 133.7 (2 C-4', s).

The syrup was applied to a $\mathrm{SiO}_{2}$ column $(2.5 \times 25 \mathrm{~cm})$ and eluted with $\mathrm{CH}_{2} \mathrm{Cl}_{2}$. Fractions containing the monotosylate 5 as the major product were combined and evaporated to give an oil $(5.1 \mathrm{~g}) .{ }^{1} \mathrm{H}$ NMR: $\delta 5.71\left(\mathrm{H}-1, \mathrm{~d}, J_{1,2}=\right.$ $3.5 \mathrm{~Hz}), 4.43\left(\mathrm{H}-2, \mathrm{~d}, J_{2,3}=0 \mathrm{~Hz}\right), 3.75\left(\mathrm{H}-3, \mathrm{~d}, J_{3,4}=3.0\right.$ $\mathrm{Hz}), 4.12\left(\mathrm{H}-4, \mathrm{dd}, J_{4.5}=7.9 \mathrm{~Hz}\right), 4.25(\mathrm{H}-5, \mathrm{~m}), 4.01$ and $4.09\left(2 \mathrm{H}-6, \mathrm{dd}, J_{\text {gem }}=8.2 \mathrm{~Hz}\right), 3.35\left(3 \mathrm{H}, \mathrm{OCH}_{3}, \mathrm{~s}\right), 1.31[3$ $\mathrm{H}, \mathrm{CH}_{3}$, isopropyl (exo), s], $1.47\left[3 \mathrm{H}, \mathrm{CH}_{3}\right.$, isopropyl (endo), s], $7.68\left(2 \mathrm{H}, \mathrm{H}-2^{\prime}, \mathrm{H}-6^{\prime}, \mathrm{d}, J=8.1 \mathrm{~Hz}\right), 7.26(2 \mathrm{H}$, H-3', H-5', d), 2.42 (3 H, $\mathrm{CH}_{3}$, tos). ${ }^{13} \mathrm{C}$ NMR: $\delta 105.0$ (C-1, d), 81.4 (C-2, d), 84.0 (C-3, d), 79.0 (C-4, d), 67.2 (C-5, d), $72.2(\mathrm{C}-6, \mathrm{t}), 111.7(\mathrm{ketal} \mathrm{C}, \mathrm{s}), 57.7\left(\mathrm{OCH}_{3}, \mathrm{q}\right)$, 26.1 and $26.6\left(2 \times \mathrm{CH}_{3}\right.$, isopropyl, q), $21.4\left(\mathrm{CH}_{3}\right.$, tos, q), $144.7\left(\mathrm{C}^{\prime}-1, \mathrm{~s}\right), 129.7,127.8\left(\mathrm{C}^{\prime}-2, \mathrm{~s}, \mathrm{C}^{\prime} 3, \mathrm{~s}\right)$ and 132.7 $\left(C^{\prime}-4, s\right)$.

5,6-Anhydro-1,2-O-isopropylidene-3-O-methyl- $\alpha$-D-glucofuranose (3) from 5. A solution of sodium methoxide prepared from $\mathrm{Na}(0.03 \mathrm{~g}, 1.3 \mathrm{mmol})$ and dry $\mathrm{MeOH}(1.5 \mathrm{ml})$ was added with stirring to a suspension of $5(0.34 \mathrm{~g}, 0.9$ mmol) in dry $\mathrm{CHCl}_{3}(3 \mathrm{ml})$. Stirring was continued for 15 min, $\mathrm{H}_{2} \mathrm{O}(10 \mathrm{ml})$ was added and the water layer was extracted with $\mathrm{CHCl}_{3}(2 \times 50 \mathrm{ml})$. Work-up yielded $3(183$ $\mathrm{mg}, 0.84 \mathrm{mmol}, 93 \%$ ) as a colourless liquid. NMR spectra were identical with those of 3 obtained above.

6-Deoxy-1,2-O-isopropylidene-3-O-methyl-a-D-hexofuranos-5-ulose (7). A Grignard reagent was prepared from $\mathrm{CH}_{3} \mathrm{I}(23.4 \mathrm{ml}, 0.38 \mathrm{~mol})$ in dry $\mathrm{Et}_{2} \mathrm{O}(100 \mathrm{ml})$ and $\mathrm{Mg}(8.2$ $\mathrm{g}, 0.34 \mathrm{~mol})$ in dry $\mathrm{Et}_{2} \mathrm{O}(200 \mathrm{ml})$. The mixture was cooled to $4{ }^{\circ} \mathrm{C}$, and a solution of $2(41 \mathrm{~g}, 0.2 \mathrm{~mol})$ in dry $\mathrm{Et}_{2} \mathrm{O}(100$ $\mathrm{ml}$ ) was added dropwise with stirring. After an additional hour of stirring at $20^{\circ} \mathrm{C}$, the reaction mixture was neutralized with sat. $\mathrm{NH}_{4} \mathrm{Cl}$, acidified with $1 \mathrm{M} \mathrm{HCl}$ and extracted with $\mathrm{Et}_{2} \mathrm{O}(3 \times 200 \mathrm{ml})$. Work-up yielded a mixture of 6deoxy-1,2-O-isopropylidene-3- $O$-methyl- $\alpha$-D-glucofuranose and 6-deoxy-1,2- $O$-isopropylidene-3- $O$-methyl- $\beta$-L-idofuranose $(40.5 \mathrm{~g}, 0.19 \mathrm{mmol}, 92 \%)$ in the ratio $37: 63$. To a vigorously stirred mixture of $\mathrm{RuO}_{2}(3.35 \mathrm{~g}, 25.2 \mathrm{mmol})$ and $\mathrm{K}_{2} \mathrm{CO}_{3}(14.3 \mathrm{~g}, 100 \mathrm{mmol})$ in $\mathrm{CH}_{2} \mathrm{Cl}_{2}(500 \mathrm{ml})$, was added dropwise a solution of $\mathrm{NaIO}_{4}(110 \mathrm{~g}, 0.51 \mathrm{~mol})$ in $\mathrm{H}_{2} \mathrm{O}(500$ $\mathrm{ml}$ ). After an additional $0.5 \mathrm{~h}$ of stirring, the above mixture of alcohols $(40 \mathrm{~g}, 0.18 \mathrm{~mol})$ in $\mathrm{CHCl}_{3}(130 \mathrm{ml})$ was added, and the reaction mixture was stirred for $48 \mathrm{~h}$. Excess oxidation agent was removed by addition of 2-propanol $(4 \mathrm{ml})$ and the precipitate was filtered. The crude product was extracted with $\mathrm{CHCl}_{3}(3 \times 40 \mathrm{ml})$ and work-up yielded 7 ( $33 \mathrm{~g}, 0.15 \mathrm{~mol}, 85 \%$ ) as a yellow syrup. ${ }^{1} \mathrm{H}$ NMR: $\delta 6.05$ $\left(\mathrm{H}-1, \mathrm{~d}, J_{1,2}=3.6 \mathrm{~Hz}\right), 4.59\left(\mathrm{H}-2, \mathrm{~d}, J_{2,3}=0 \mathrm{~Hz}\right), 4.03$ $\left(\mathrm{H}-3, \mathrm{~d}, J_{3,4}=3.7 \mathrm{~Hz}\right), 4.58(\mathrm{H}-4, \mathrm{~d}), 2.23(3 \mathrm{H}, \mathrm{C} 5-\mathrm{Me}, \mathrm{s})$, $3.36\left(3 \mathrm{H}, \mathrm{OCH}_{3}, \mathrm{~s}\right), 1.49\left[3 \mathrm{H}, \mathrm{CH}_{3}\right.$, isopropyl (endo), s], $1.34\left[3 \mathrm{H}, \mathrm{CH}_{3}\right.$, isopropyl (exo), s]. ${ }^{13} \mathrm{C} \mathrm{NMR:} \delta 105.6(\mathrm{C}-1$, d), 80.9 (C-2, d), 85.6 (C-3, d), 85.1 (C-4, d), 206.2 (C-5, s), $112.0(\mathrm{ketal} \mathrm{C}, \mathrm{S}), 57.9\left(\mathrm{OCH}_{3}, \mathrm{q}\right), 27.9(\mathrm{C}-6, \mathrm{q}), 26.6$ and $26.1\left(2 \times \mathrm{CH}_{3}\right.$, isopropyl, q).

Addition of diazomethane to 6-deoxy-1,2-O-isopropylidene-3-O-methyl- $\alpha$-D-xylo-hexofuranos-5-ulose (7). A 0.44 molar solution of $\mathrm{CH}_{2} \mathrm{~N}_{2}$ in $\mathrm{Et}_{2} \mathrm{O}(65 \mathrm{ml}, 28.4 \mathrm{mmol})$ was added dropwise to stirred 6 ( $2.84 \mathrm{~g}, 13.1 \mathrm{mmol})$ in $\mathrm{Et}_{2} \mathrm{O}$ (30 $\mathrm{ml}$ ). An additional $24 \mathrm{~h}$ of stirring and evaporation of the solvent under reduced pressure gave a dark yellow liquid $(3.0 \mathrm{~g})$. TLC showed four spots and the major one $(60 \%$, $R_{\mathrm{f}}=0.75$ in $\mathrm{Et}_{2} \mathrm{O}$ ) was shown (GLC) to be a mixture of the epimeric epoxides 8 and $9,(8: 9=40: 60)$. When the addition reaction was performed in a solvent consisting of $25 \%$ $\mathrm{MeOH}$ and $75 \% \mathrm{Et}_{2} \mathrm{O}$, the ratio 8:9 was 55:45 (GLC). The reaction mixture was applied to a column $\left(\mathrm{SiO}_{2}\right)$ and eluted with $\mathrm{Et}_{2} \mathrm{O}$. The combined fractions containing 8 and 9 were applied to another column $\left(\mathrm{SiO}_{2}\right)$ and eluted with hexane: $\mathrm{Et}_{2} \mathrm{O}=70: 30$ and 65 fractions $(50 \mathrm{ml})$ were collected. Fractions $42-46(94.3 \mathrm{mg}, 3 \%)$ contained 5,6-anhydro-3- $O, 5$ - $C$-dimethyl-1,2- $O$-isopropylidene- $\beta$-L-idofuranose (8). Calcd. for $\mathrm{C}_{11} \mathrm{H}_{18} \mathrm{O}_{5}: 230.1154$. Obs. $M^{\cdot+}=$ 230.1146. $\mathrm{m} / \mathrm{z}(\%)$ : $230(0.1), 215$ (5), 145 (10), 144 (17), 141 (12), 87 (22), $86(31), 85(91), 83(25), 59(70), 56(73)$, $57(21), 43$ (100). ${ }^{1} \mathrm{H}$ NMR: $5.89\left(\mathrm{H}-1, \mathrm{~d}, J_{1,2}=3.4 \mathrm{~Hz}\right)$, $4.52\left(\mathrm{H}-2, \mathrm{~d}, J_{2,3}=0 \mathrm{~Hz}\right), 3.74\left(\mathrm{H}-3, \mathrm{~d}, J_{3,4}=3.9 \mathrm{~Hz}\right), 2.53$ [H-6a ( cis $\mathrm{CH}_{3}$ ), d, $\left.J_{\text {gem }}=5.1 \mathrm{~Hz}\right], 2.79\left[\mathrm{H}-6 \mathrm{~b}\right.$ (trans $\mathrm{CH}_{3}$ ), d], $3.42\left(3 \mathrm{H}, \mathrm{OCH}_{3}, \mathrm{~s}\right), 1.46\left[3 \mathrm{H}, \mathrm{CH}_{3}\right.$, isopropyl (endo), s], $1.38\left[3 \mathrm{H}, \mathrm{CH}_{3}\right.$, isopropyl (exo), s], $1.38(3 \mathrm{H}, \mathrm{C} 5-\mathrm{Me}$, s). ${ }^{13} \mathrm{C}$ NMR: $\delta 105.1$ (C-1, d), 82.1 (C-4, d), 82.3 (C-2, d), 85.8 (C-3, d), 55.1 (C-5, s), 49.7 [C-5 (epoxide), t], 111.6 (ketal C, s), $57.7\left(\mathrm{OCH}_{3}, \mathrm{q}\right), 18.4$ (C5-Me, q), 26.3 and $26.8\left(2 \times \mathrm{CH}_{3}\right.$, isopropyl, q). Fractions $19-27(0.26 \mathrm{~g}$, $8.6 \%$ ) contained 5,6-anhydro-5- $C$-methyl-1,2- $O$-isopropylidene-D-glucofuranose (9). MS: Calcd. for $\mathrm{C}_{11} \mathrm{H}_{18} \mathrm{O}_{5}$ : 230.1154. Obs. $M^{\bullet+}$ 230.1146. $\mathrm{m} / z$ (\%): $230(0.4), 215$ (7), 
145 (14), 144 (23), 87 (25), 85 (37), 85 (86), 83 (26.2), 59 (79), 58 (72), 43 (100). ${ }^{1} \mathrm{H}$ NMR: $\delta 5.88\left(\mathrm{H}-1, \mathrm{~d}, J_{1,2}=3.4\right.$ $\mathrm{Hz}), 4.58\left(\mathrm{H}-2, \mathrm{~d}, J_{2,3}=0 \mathrm{~Hz}\right), 3.77\left(\mathrm{H}-3, J_{3,4}=3.4 \mathrm{~Hz}\right)$, $4.24(\mathrm{H}-4, \mathrm{~d}), 2.75$ [H6a (cis $\left.\mathrm{CH}_{3}\right), \mathrm{d}, J_{\text {gem }}=4.9 \mathrm{~Hz}$ ], 3.03 [H 6b (trans $\left.\left.\mathrm{CH}_{3}\right), \mathrm{d}\right], 3.43\left(3 \mathrm{H}, \mathrm{OCH}_{3}, \mathrm{~s}\right), 1.49\left[3 \mathrm{H}, \mathrm{CH}_{3}\right.$, isopropyl (endo), s], $1.32\left[3 \mathrm{H}, \mathrm{CH}_{3}\right.$, isopropyl (exo), s], $1.38\left[3 \mathrm{H}, \mathrm{CH}_{3}(\mathrm{C} 5-\mathrm{Me}), \mathrm{s}\right] .{ }^{13} \mathrm{C}$ NMR: $\delta 105.1(\mathrm{C}-1, \mathrm{~d})$, 80.0 (C-4, d) 81.4 (C-2, d), 84.9 (C-3, d), 55.5 (C-5, s), 51.6 [C-6 (epoxide), t], 111.8 (ketal C, s), 18.8 (C5-Me, q), 26.6 and $26.9\left(2 \times \mathrm{CH}_{3}\right.$, isopropyl, q), $57.8\left(\mathrm{OCH}_{3}, \mathrm{q}\right)$.

\section{1,2-O-Isopropylidene-3-O,5-C-dimethyl- $\beta$-L-idofuranose} (10). Compound 10 was made by adding $0.5 \mathrm{M} \mathrm{NaOH}(5$ $\mathrm{ml})$ to $8(46 \mathrm{mg}, 0.2 \mathrm{mmol})$. After being stirred at $60^{\circ} \mathrm{C}$ for $6 \mathrm{~h}$, the reaction mixture was neutralized with $1 \mathrm{M} \mathrm{HCl} / \mathrm{sat}$. $\mathrm{NH}_{4} \mathrm{Cl}$ and extracted with $\mathrm{CHCl}_{3}(3 \times 30 \mathrm{ml})$. Work-up gave $10(48 \mathrm{mg}, 0.19 \mathrm{mmol})$ as a colourless liquid: $[\alpha]_{\mathrm{D}}=$ $-39.6^{\circ}\left(\mathrm{CHCl}_{3}, c=2.4\right)$; MS: Calcd. for $\mathrm{C}_{11} \mathrm{H}_{19} \mathrm{O}_{6}(M-1)$ : 247.1182. Obs. $(M-1)$ 247.1181. $m / z$ (\%): 233 (2.4), 217 (24), 185 (5), 173 (10), 159 (19), 142 (8), 114 (13), 113 (51), 101 (52), 85 (67), 59 (75), 58 (66), 43 (100). ${ }^{1} \mathrm{H}$ NMR: $\delta$ $6.00\left(\mathrm{H}-1, \mathrm{~d}, J_{1,2}=3.9 \mathrm{~Hz}\right), 4.58\left(\mathrm{H}-2, \mathrm{~d}, J_{2,3}=0 \mathrm{~Hz}\right), 4.04$ $\left(\mathrm{H}-3, \mathrm{~d}, J_{3.4}=3.4 \mathrm{~Hz}\right), 3.92\left(3 \mathrm{H}, \mathrm{OCH}_{3}, \mathrm{~s}\right), 1.30\left[3 \mathrm{H}, \mathrm{CH}_{3}\right.$ (C5-Me), s], 1.50 [3 H, $\mathrm{CH}_{3}$, isopropyl (endo), s], 1.33 [3 $\mathrm{H}, \mathrm{CH}_{3}$, isopropyl (exo), s]. ${ }^{13} \mathrm{C}$ NMR: $\delta 104.9(\mathrm{C}-1, \mathrm{~d})$, 81.0 (C-2, d), 86.1 (C-3, d), 80.6 (C-4, d), 72.8 (C-5, s), $68.1(\mathrm{C}-6, \mathrm{t}), 111.7$ (ketal C, s), $57.5\left(\mathrm{OCH}_{3}, \mathrm{q}\right), 22.4$ (C5-Me, q), 26.7 and $26.1\left(2 \times \mathrm{CH}_{3}\right.$, isopropyl, q).

1,2-O-Isopropylidene-3-O,5-C-dimethyl- $\alpha$-D-glucofuranose (11). Compound 11 was made by adding $0.5 \mathrm{M} \mathrm{NaOH}(12$ $\mathrm{ml})$ to $9(0.13 \mathrm{~g}, 0.57 \mathrm{mmol})$. After $3 \mathrm{~h}$ of stirring at $60^{\circ} \mathrm{C}$, the reaction mixture was neutralized with $1 \mathrm{M} \mathrm{HCl} / \mathrm{sat}$. $\mathrm{NH}_{4} \mathrm{Cl}$ and extracted with $\mathrm{CHCl}_{3}(3 \times 75 \mathrm{ml})$. Work-up gave $11(0.13 \mathrm{~g}, 0.53 \mathrm{mmol}, 92 \%)$ as a colourless liquid: $[\alpha]_{\mathrm{D}}=-45.0^{\circ}\left(\mathrm{CHCl}_{3}, c=2.86\right)$; MS: Calcd. for $\mathrm{C}_{11} \mathrm{H}_{19} \mathrm{O}_{3}$ $(M-1)=247.1182$. Obs. $\left(M^{\bullet}+-1\right)=247.1181 . \mathrm{m} / z(\%)$ : $233(2), 217(17), 185(3), 173(7), 159(6), 142(9), 114(13)$, 113 (47), 101 (48), 85 (69), 59 (79), 58 (60), 43 (100). ${ }^{1} \mathrm{H}$ NMR: $\delta \cdot 5.9\left(\mathrm{H}-1, \mathrm{~d}, J_{1,2}=3.9 \mathrm{~Hz}\right), 4.58(\mathrm{H}-2, \mathrm{~d}), 3.97$ $\left(\mathrm{H}-3, J_{3.4}=3.4 \mathrm{~Hz}\right), 3.90(\mathrm{H}-4, \mathrm{~d}), 3.52$ and $3.59(\mathrm{H}-6 \mathrm{a}$ and $\left.\mathrm{H}-6 \mathrm{~b}, J_{\text {gem }}=7.8 \mathrm{~Hz}\right), 3.43\left(3 \mathrm{H}, \mathrm{OCH}_{3}, \mathrm{~s}\right), 1.28\left[3 \mathrm{H}, \mathrm{CH}_{3}\right.$ (C5-Me), s], 1.49 [3 H, $\mathrm{CH}_{3}$, isopropyl (endo), s], 1.33 [3 $\mathrm{H}, \mathrm{CH}_{3}$, isopropyl (exo), s], ${ }^{13} \mathrm{C}$ NMR: $\delta 103.9$ (C-1, d), 80.8 (C-4, d), 85.2 (C-3, d), 82.0 (C-2, d), 73.3 (C-5, s), $68.3(\mathrm{C}-6, \mathrm{t}) 111.2$ ketal $\mathrm{C}, \mathrm{s}), 56.9\left(\mathrm{OCH}_{3}, \mathrm{q}\right), 21.8$ (C5-Me, q), 26.3 and $25.8\left(2 \times \mathrm{CH}_{3}\right.$, isopropyl, q).

1,6-Anhydro-3-O,5-C-dimethyl- $\beta$-L-idopyranose (12). Compound 12 was made by hydrolysing $10(40 \mathrm{mg}, 0.16$ mmol) with $2 \% \mathrm{H}_{2} \mathrm{SO}_{4}(5 \mathrm{ml})$. After being stirred for $4 \mathrm{~h}$ at $95^{\circ} \mathrm{C}$, the reaction mixture was neutralized with $\mathrm{BaCO}_{3}$, filtered and extracted with $\mathrm{CHCl}_{3}(2 \times 10 \mathrm{ml})$. Work-up yielded $12(31 \mathrm{mg}, 14.9 \mathrm{mmol}, 92.6 \%)$ as white crystals. Recrystallization from $\mathrm{CHCl}_{3}$ yielded colourless needles, m.p. $138.5-139^{\circ} \mathrm{C} . R_{\mathrm{f}}$ (TLC) $=0.58$ in acetone. ${ }^{1} \mathrm{H}$ NMR: $\delta$ $5.25\left(\mathrm{H}-1, \mathrm{~d}, J_{1,2}=1.9 \mathrm{~Hz}\right), 3.56\left(\mathrm{H}-2, \mathrm{dt}, J_{2,3}=8.4 \mathrm{~Hz}\right)$,
$3.25\left(\mathrm{H}-3, \mathrm{t}, J_{3,4}=8.4 \mathrm{~Hz}\right), 3.52\left(\mathrm{H}-4, \mathrm{dd}, J_{4,6 \mathrm{~b}}=1.4 \mathrm{~Hz}\right)$, 3.38 [H-6b (exo), dd, $J_{\text {gem }}=8.1 \mathrm{~Hz}$ ], 4.02 [H-6a (endo), d], $2.68(\mathrm{H}-\mathrm{OH}-4, \mathrm{~d}, J=3.3 \mathrm{~Hz}), 2.26(\mathrm{H}-\mathrm{OH}-2, \mathrm{~d}, J=9.3$ $\mathrm{Hz}), 1.42\left(3 \mathrm{H}, \mathrm{CH}_{3}, \mathrm{C} 5-\mathrm{Me}, \mathrm{s}\right), 3.56\left(3 \mathrm{H}, \mathrm{OCH}_{3}, \mathrm{~s}\right) .{ }^{13} \mathrm{C}$ NMR: $\delta 102.0$ (C-1, d), 86.2 (C-3, d), 80.8 (C-5, s), 75.4 $(\mathrm{C}-2, \mathrm{~d}), 75.2$ (C-4, d), 69.9 (C-6, t), $60.1\left(\mathrm{OCH}_{3}, \mathrm{q}\right), 18.5$ (C5-Me, q). MS: Calcd. for $\mathrm{C}_{7} \mathrm{H}_{10} \mathrm{O}_{4}\left(M-\mathrm{HOCH}_{3}\right)$ : 158.0573. Obs. $(M-32)=158.0573 . m / z(\%): 158(2), 131$ (4), 117 (63), 101 (4), 85 (5), 75 (5), 74 (100), 73 (12), 71 (16), 43 (42).

3-O, 5-C-Dimethyl-D-glucopyranose (13). To a mixture of 10 and 11 (157 mg, $0.63 \mathrm{mmol}, 10: 11=53: 47$, GLC) was added $0.8 \% \mathrm{H}_{2} \mathrm{SO}_{4}(10 \mathrm{ml})$. After $13 \mathrm{~h}$ at $80^{\circ} \mathrm{C}$, neutralization with $\mathrm{BaCO}_{3}$ and filtration, the filtrate was extracted with $\mathrm{CHCl}_{3}(3 \times 20 \mathrm{ml})$. Usual work-up yielded a colourless syrup ( $84 \mathrm{mg}, 0.40 \mathrm{mmol}, 63.5 \%$ ). PLC (acetone) gave three fractions: The first fraction was shown to be 12 (42 $\left.\mathrm{mg}, R_{\mathrm{f}}=0.58\right)$, the second fraction $\left(8.9 \mathrm{mg}, R_{\mathrm{f}}=0.47\right)$ contained a compound only consistent with 1,6 -anhydro-3$O, 5-C$-dimethyl- $\beta$-D-glucopyranose (14) and the third fraction contained $13\left(26 \mathrm{mg}, R_{\mathrm{f}}=0.24\right)$. The anomeric ratio, $\alpha: \beta$ was $15: 85$ (400 $\mathrm{MHz}^{1} \mathrm{H}$ NMR in $\left.\mathrm{D}_{2} \mathrm{O}\right)$. NMR data for the $\beta$-anomer: ${ }^{1} \mathrm{H}$ NMR $\left(\mathrm{D}_{2} \mathrm{O}\right): \delta 4.78\left(\mathrm{H}-1, \mathrm{~d}, J_{1,2}=7.8\right.$ $\mathrm{Hz}), 3.24\left(\mathrm{H}-2\right.$, dd, $\left.J_{2,3}=9.3 \mathrm{~Hz}\right), 3.32\left(\mathrm{H}-3, \mathrm{t}, J_{3,4}=9.3\right.$ $\mathrm{Hz}), 3.65(\mathrm{H}-4, \mathrm{~d}), 3.58$ and 3.54 [H-6a and $\mathrm{H}-6 \mathrm{~b}, \mathrm{~d}, J_{\text {gem }}=$ $11.5 \mathrm{~Hz}$ ], $1.23\left[3 \mathrm{H}, \mathrm{CH}_{3}(\mathrm{C} 5-\mathrm{Me}), \mathrm{s}\right], 3.72\left(3 \mathrm{H}, \mathrm{OCH}_{3}, \mathrm{~s}\right)$, ${ }^{13} \mathrm{C}$ NMR $\left(\mathrm{D}_{2} \mathrm{O}\right): \delta 94.1(\mathrm{C}-1, \mathrm{~d}), 77.3(\mathrm{C}-2, \mathrm{~d}), 85.2(\mathrm{C}-3$, d), $72.3(\mathrm{C}-4, \mathrm{~d}), 78.3(\mathrm{C}-5, \mathrm{~s}), 68.6(\mathrm{C}-6, \mathrm{t}), 14.9$ (C5-Me, q), $61.2\left(\mathrm{OCH}_{3}, \mathrm{q}\right)$.

1,6-Anhydro-3-O,5-C-dimethyl- $\beta$-D-glucopyranose (14). To 11 was added $2 \% \mathrm{H}_{2} \mathrm{SO}_{4}(12 \mathrm{ml})$ and the mixture was stirred for $4 \mathrm{~h}$ at $95^{\circ} \mathrm{C}$. Neutralization with $\mathrm{BaCO}_{3}$, filtration and evaporation of the solvent yielded a mixture of 13 and 14 (total $94.5 \mathrm{mg}$ ). PLC of the reaction mixture using acetone as the eluent yielded $13\left(43.7 \mathrm{mg}, R_{\mathrm{f}}=0.24\right)$ and $14\left(39 \mathrm{mg}, R_{\mathrm{f}}=0.47\right)$. Data for 14: ${ }^{1} \mathrm{H}$ NMR: $\delta 5.45$ (H-1, br s), 3.65 (H-2, br s), $3.46\left(\mathrm{H}-3, \mathrm{~d}, J_{3,4}<1 \mathrm{~Hz}\right), 3.53$ $(\mathrm{H}-4, \mathrm{~d}), 4.19$ [H-6a (endo), d, $\left.J_{\text {gem }}=7.1 \mathrm{~Hz}\right], 3.43$ [H-6b (exo), d], $3.45\left(3 \mathrm{H}, \mathrm{OCH}_{3}, \mathrm{~s}\right), 2.65(1 \mathrm{H}, \mathrm{OH}-4, \mathrm{~s}), 2.20$ (1 $\mathrm{H}, \mathrm{OH}-2, \mathrm{~s}), 1.47$ (3 H, $\left.\mathrm{CH}_{3}, \mathrm{C} 5-\mathrm{Me}, \mathrm{s}\right),{ }^{13} \mathrm{C}$ NMR (CD $\left.{ }_{3} \mathrm{OD}\right): \delta 103.8$ (C-1, d), 71.3 (C-2, d), 85.0 (C-3, d), $71.1(\mathrm{C}-4, \mathrm{~d}), 81.1(\mathrm{C}-5, \mathrm{~s}), 67.9(\mathrm{C}-6, \mathrm{t}), 57.9\left(\mathrm{OCH}_{3}, \mathrm{q}\right)$, 18.6 (C5-Me, q).

1,2-O-Isopropylidene-3-O-methyl-5-C-phenyl- $\alpha$-D-xylopentofuranos-5-ulose (15). A Grignard reaction was performed by dropwise addition of a solution of $\mathrm{PhI}(6.12 \mathrm{~g}$, $3.4 \mathrm{ml}, 29.9 \mathrm{mmol})$ in dry $\mathrm{Et}_{2} \mathrm{O}(12 \mathrm{ml})$ to a suspension of $\mathrm{Mg}(0.34 \mathrm{~g}, 14 \mathrm{mmol})$ in dry $\mathrm{Et}_{2} \mathrm{O}(5 \mathrm{ml})$. After the reaction mixture had been refluxed for $0.5 \mathrm{~h}, 2(3.0 \mathrm{~g}, 14.9 \mathrm{mmol})$ in dry $\mathrm{Et}_{2} \mathrm{O}(15 \mathrm{ml})$ was added dropwise. Stirring at room temperature was maintained for $18 \mathrm{~h}$ before acidification with $1 \mathrm{M} \mathrm{HCl}$ and extraction with $\mathrm{CHCl}_{3}(3 \times 50 \mathrm{ml})$. Work-up yielded a brown syrup $(4.1 \mathrm{~g}, 14.6 \mathrm{mmol}, 98.2 \%)$ which was oxidized without further purification. To a 
vigorously stirred mixture of $\mathrm{RuO}_{2}(0.35 \mathrm{~g}, 2.63 \mathrm{mmol})$, $\mathrm{K}_{2} \mathrm{CO}_{3}(1.43 \mathrm{~g}, 0.01 \mathrm{mmol})$ and $\mathrm{CH}_{2} \mathrm{Cl}_{2}(50 \mathrm{ml})$ was added $\mathrm{NaIO}_{4}(9 \mathrm{~g}, 42 \mathrm{mmol})$ in $\mathrm{H}_{2} \mathrm{O}(50 \mathrm{ml})$. After addition of the previously mentioned Grignard product in $\mathrm{CHCl}_{3}(10 \mathrm{ml})$, stirring was continued for an additional $48 \mathrm{~h}$. In order to speed up the reaction, additional $\mathrm{NaIO}_{4}(5 \mathrm{~g}, 23.4 \mathrm{mmol})$ and $\mathrm{RuO}_{2}(0.2 \mathrm{~g}, 1.5 \mathrm{mmol})$ were added. When the reaction was complete, excess oxidant was reduced by addition of 2-propanol. Work-up yielded $15(3.9 \mathrm{~g}, 14 \mathrm{mmol}, 96 \%)$ as a dark syrup. Crystallization from $\mathrm{MeOH}$ gave colourless crystals, m.p. $122-123^{\circ} \mathrm{C} .{ }^{1} \mathrm{H}$ NMR: $\delta 6.14(\mathrm{H}-1, \mathrm{~d}$, $\left.J_{1,2}=3.9 \mathrm{~Hz}\right), 4.64\left(\mathrm{H}-2, \mathrm{~d}, J_{2,3}=0 \mathrm{~Hz}\right), 4.20\left(\mathrm{H}-3, \mathrm{~d}, J_{3,4}\right.$ $=3.9 \mathrm{~Hz}), 5.54(\mathrm{H}-4, \mathrm{~d}), 3.18\left(3 \mathrm{H}, \mathrm{OCH}_{3}, \mathrm{~s}\right), 1.56[3 \mathrm{H}$, $\mathrm{CH}_{3}$, isopropyl(endo), s], 1.37 [ $3 \mathrm{H}, \mathrm{CH}_{3}$, isopropyl(exo), s], $7.96(2 \mathrm{H}-o, \mathrm{~d}), 7.48(2 \mathrm{H}-m, \mathrm{t}), 7.53(1 \mathrm{H}-p, \mathrm{t}) .{ }^{13} \mathrm{C}$ NMR: $\delta 105.4$ (C-1, d), 81.5 (C-2, d), 86.2 (C-3, d), 83.5 $(\mathrm{C}-4, \mathrm{~d}), 194.0(\mathrm{C}-5, \mathrm{~s}), 26.3$ and $27.0\left(2 \times \mathrm{CH}_{3}\right.$, isopropyl, q), $58.1\left(\mathrm{OCH}_{3}, \mathrm{q}\right), 136.0(\mathrm{C}-1, \mathrm{~s}), 133.2(\mathrm{C}-4, \mathrm{~d}), 128.1$ and 128.5 (C-2, C-3, C-5 and C-6, all d).

5,6-Anhydro-1,2-O-isopropylidene-3-O-methyl-5-C-phenyl$\alpha$-D-glucofuranose (17) and 5,6-anhydro-1,2-O-isopropylidene-3-O-methyl-5-C-phenyl- $\beta$-L-idofuranose (16). To a solution of $15(100 \mathrm{mg}, 0.36 \mathrm{mmol})$ in $\mathrm{Et}_{2} \mathrm{O}(5 \mathrm{ml})$, saturated with $\mathrm{H}_{2} \mathrm{O}$, was added dropwise a solution of $\mathrm{CH}_{2} \mathrm{~N}_{2}(0.8 \mathrm{mmol})$ in $\mathrm{Et}_{2} \mathrm{O}(4 \mathrm{ml})$. After $12 \mathrm{~h}$ at room temperature the reaction mixture was concentrated under reduced pressure to yield a thin, colourless syrup $(103 \mathrm{mg})$. TLC of the reaction mixture showed seven spots of which the epimeric epoxides were the major components $(60 \%)$ and the isomeric benzyl ketone a minor component $(10 \%)$ ( ${ }^{1} \mathrm{H}$ NMR). The reaction mixture $(67 \mathrm{mg})$ was applied to PLC plates and eluted with EtOAc: $\mathrm{CH}_{2} \mathrm{Cl}_{2}=30: 70$. Fraction $3\left(R_{\mathrm{f}}=0.63\right)$ and fraction $4\left(R_{\mathrm{f}}=0.68\right)$ contained 17 and 16, respectively. The ratio $17: 16$ was $11: 89$ (GLC and ${ }^{1} \mathrm{H}$ NMR). ${ }^{1} \mathrm{H}$ NMR (17): $\delta 5.95\left(\mathrm{H}-1, \mathrm{~d}, J_{1,2}=3.7 \mathrm{~Hz}\right)$, $4.23\left(\mathrm{H}-2, \mathrm{~d}, J_{2,3}=0 \mathrm{~Hz}\right), 3.81\left(\mathrm{H}-3, \mathrm{~d}, J_{3,4}=3.2 \mathrm{~Hz}\right), 4.61$ $(\mathrm{H}-4, \mathrm{~d}), 2.85[\mathrm{H}-6 \mathrm{~b}$ (trans $\left.\mathrm{Ph}), \mathrm{d}, J_{\text {gem }}=7.0 \mathrm{~Hz}\right], 2.43$ [H-6a (cis Ph), d], $3.45\left(3 \mathrm{H}, \mathrm{OCH}_{3}, \mathrm{~s}\right), 1.55\left[3 \mathrm{H}, \mathrm{CH}_{3}\right.$, isopropyl(endo), s], $1.32\left[3 \mathrm{H}, \mathrm{CH}_{3}\right.$, isopropyl(exo), s], 7.20-7.50 (5 H, Ph, m). ${ }^{1} \mathrm{H}$ NMR (16): $\delta 5.99$ (H-1, d, $J_{1,2}=$ $3.8 \mathrm{~Hz}), 4.58\left(\mathrm{H}-2, \mathrm{~d}, J_{2,3}=0 \mathrm{~Hz}\right), 3.59\left(\mathrm{H}-3, \mathrm{~d}, J_{3,4}=3.3\right.$ $\mathrm{Hz}), 4.87(\mathrm{H}-4, \mathrm{~d}), 3.28$ [H-6b (trans $\mathrm{Ph}), \mathrm{d}, J_{\text {gem }}=7.2 \mathrm{~Hz}$, 2.58 [H-6a (cis $\mathrm{Ph}), \mathrm{d}], 3.26\left(3 \mathrm{H}, \mathrm{OCH}_{3}, \mathrm{~s}\right), 1.55[3 \mathrm{H}$, $\mathrm{CH}_{3}$, isopropyl(endo), s], $1.34\left[3 \mathrm{H}, \mathrm{CH}_{3}\right.$, isopropyl(exo), s], 7.20-7.50 (5 H, Ph, m). ${ }^{13} \mathrm{C} \mathrm{NMR} \mathrm{(16):} \delta 104.9$ (C-1, d), 81.7 (C-2, d), 84.3 (C-3, d), 78.6 (C-4, d), 58.1 (C-5, s), $50.1(\mathrm{C}-6, \mathrm{t}), 57.8\left(\mathrm{CH}_{3}, \mathrm{q}\right), 111.6($ ketal $\mathrm{C}, \mathrm{s}), 26.1$ and $26.9\left(2 \times \mathrm{CH}_{3}\right.$, isopropyl, q), 126.4, 127.8, 128.3, 129.9 and $138.1(\mathrm{Ph})$.

1,2-O-Isopropylidene-3-O-methyl-5-C-phenyl- $\alpha$-D-glucofuranose (19) and 1,2-O-isopropylidene-3-O-methyl-5-Cphenyl- $\beta$-L-idofuranose (18). To a mixture of 17 and 16 (50 $\mathrm{mg}, 0.17 \mathrm{mmol}, 17: 16=11: 89)$ was added $0.5 \mathrm{M} \mathrm{NaOH}$ $(7 \mathrm{ml})$. After being stirred at $60^{\circ} \mathrm{C}$ for $12 \mathrm{~h}$, the reaction mixture was neutralized with $1 \mathrm{M} \mathrm{HCl} / \mathrm{sat}$. $\mathrm{NH}_{4} \mathrm{Cl}$ and extracted with $\mathrm{CHCl}_{3}(3 \times 5 \mathrm{ml})$. Work-up yielded a mixture of 19 and $18(49 \mathrm{mg}, 0.16 \mathrm{mmol}, 93 \%, 19: 18=11: 89)$ as a colourless syrup. ${ }^{1} \mathrm{H}$ NMR (18): $\delta 6.05\left(\mathrm{H}-1, \mathrm{~d}, J_{1,2}=3.0\right.$ $\mathrm{Hz}), 4.43\left(\mathrm{H}-2, \mathrm{~d}, J_{2,3}=0 \mathrm{~Hz}\right), 3.43\left(\mathrm{H}-3, \mathrm{~d}, J_{3,4}=3.4 \mathrm{~Hz}\right)$, $4.56(\mathrm{H}-4, \mathrm{~d}), 4.00$ and $3.88\left(\mathrm{H}-6 \mathrm{a}\right.$ and $\mathrm{H}-6 \mathrm{~b}, \mathrm{~d}, J_{\text {gem }}=11.2$ $\mathrm{Hz}), 3.07\left(3 \mathrm{H}, \mathrm{OCH}_{3}, \mathrm{~s}\right), 1.53\left[3 \mathrm{H}, \mathrm{CH}_{3}\right.$, isopropyl(endo), s], $1.32\left[3 \mathrm{H}, \mathrm{CH}_{3}\right.$, isopropyl $($ exo $\left.), \mathrm{s}\right], 7.20-7.50(5 \mathrm{H}, \mathrm{Ph}$, $\mathrm{m}), 3.75$ and 2.28 (OH-6, OH-5). ${ }^{13} \mathrm{C}$ NMR (18): $\delta 105.4$ (C-1, d), 81.7 (C-2, d), 86.2 (C-3, d), 80.7 (C-4, d), 75.7 $(\mathrm{C}-5, \mathrm{~s}), 69.4(\mathrm{C}-6, \mathrm{t}), 57.7\left(\mathrm{OCH}_{3}, \mathrm{q}\right), 26.1$ and 26.8 $\left(2 \times \mathrm{CH}_{3}\right.$, isopropyl, q), 125.1, 127.4, 128.4, 130.7 and $142.4(\mathrm{Ph})$.

1,6-Anhydro-3-O-methyl-5-C-phenyl- $\beta$-L-idopyranose (20). Compound 20 was made by adding $0.8 \% \mathrm{H}_{2} \mathrm{SO}_{4}(15 \mathrm{ml})$ to a mixture of 19 and $18(49 \mathrm{mg}, 0.16 \mathrm{mmol}, 19: 18=11: 89)$ in $\mathrm{MeOH}(15 \mathrm{ml})$. Stirring at $85^{\circ} \mathrm{C}$ for $24 \mathrm{~h}$, neutralization with $\mathrm{BaCO}_{3}$ and work-up yielded a thin syrup (41 mg, 0.15 mmol, $96 \%$ ) consisting mainly of $20 .{ }^{1} \mathrm{H}$ NMR: $\delta 5.48$ $\left(\mathrm{H}-1, \mathrm{~d}, J_{1,2}=1.3 \mathrm{~Hz}\right), 3.74\left(\mathrm{H}-2, \mathrm{dd}, J_{2,3}=6.8 \mathrm{~Hz}\right), 3.37$ $\left(\mathrm{H}-3\right.$, dd, $\left.J_{3,4}=6.6 \mathrm{~Hz}\right), 3.65\left(\mathrm{H}-4, \mathrm{dd}, J_{4,6 \mathrm{~b}(\mathrm{exo})}=0.9 \mathrm{~Hz}\right)$, 4.58 [H-6a (endo), d, $J_{\text {gem }}=7.7 \mathrm{~Hz}$ ], 3.79 [H-6b (exo), dd], $3.66\left(3 \mathrm{H}, \mathrm{OCH}_{3}, \mathrm{~s}\right), 1.85$ and $2.28(\mathrm{OH}-4, \mathrm{OH}-2)$, 7.20-7.50 (5 H, Ph, m).

\section{References}

1. For a review on branched carbohydrates see: Grisebach, $\mathbf{H}$. and Schmid, R. Angew. Chem. 84 (1972) 192.

2. Anthonsen, T., Hagen, S., Kazi, M. A., Shah, S. W. and Tagar, S. Acta Chem. Scand., Ser. B 30 (1976) 91.

3. Kringstad, R., Singsaas, A. O., Rusten, G., Bækkemoen, G., Paulsen, B. S. and Nordal, A. Phytochemistry 19 (1980) 543.

4. Yoshimura, J. Adv. Carbohydr. Chem. 42 (1984) 69.

5. Shasha, B. S. and Doane, W. M. Carbohydr. Res. 34 (1974) 370.

6. Guthrie, R. D. Methods in Carbohydrate Research, Academic Press, New York 1962, Vol. 1, p. 432.

7. Reitsøen, B., Kilaas, L. and Anthonsen, T. Acta Chem. Scand., Ser. B 40 (1986) 440.

8. Kilaas, L. and Anthonsen, T. Acta Chem. Scand., Ser. B39 (1985) 685.

9. Hagen, S., Anthonsen, T. and Kilaas, L. Tetrahedron 35 (1979) 2583.

10. Gutsche, C. D. Org. React. 8 (1954) 367.

11. Corey, E. J. and Chaykovsky, M. J. Am. Chem. Soc. 87 (1965) 1353.

12. Stoddart, J. F. Stereochemistry of Carbohydrates, Wiley Interscience, New York 1971, p. 59.

13. Furniss, B. S., Hannaford, A. J., Smith, P. W. G. and Tatchell, A. R. Vogel's Textbook of Practical Organic Chemistry, 5th ed., Longman Scientific and Technical, Essex, UK 1989, p. 432.

Received February 7, 1992. 